\title{
을 Loss-of-function mutations in LEMD3 result in osteopoikilosis, Buschke-Ollendorff syndrome and melorheostosis
}

Jan Hellemans ${ }^{1}$, Olena Preobrazhenska ${ }^{2}$, Andy Willaert ${ }^{1}$, Philippe Debeer ${ }^{3}$, Peter C M Verdonk ${ }^{4}$, Teresa Costa ${ }^{5}$, Katrien Janssens ${ }^{6}$, Bjorn Menten ${ }^{1}$, Nadine Van Roy ${ }^{1}$, Stefan J T Vermeulen ${ }^{1}$, Ravi Savarirayan ${ }^{7}$, Wim Van Hul ${ }^{6}$, Filip Vanhoenacker ${ }^{8}$, Danny Huylebroeck ${ }^{2}$, Anne De Paepe ${ }^{1}$, Jean-Marie Naeyaert ${ }^{9}$, Jo Vandesompele ${ }^{1}$, Frank Speleman ${ }^{1}$, Kristin Verschueren ${ }^{2}$, Paul J Coucke ${ }^{1}$ \& Geert R Mortier ${ }^{1}$

Osteopoikilosis, Buschke-Ollendorff syndrome (BOS) and melorheostosis are disorders characterized by increased bone density ${ }^{1}$. The occurrence of one or more of these phenotypes in the same individual or family suggests that these entities might be allelic ${ }^{2-4}$. We collected data from three families in which affected individuals had osteopoikilosis with or without manifestations of BOS or melorheostosis. A genome-wide linkage analysis in these families, followed by the identification of a microdeletion in an unrelated individual with these diseases, allowed us to map the gene that is mutated in osteopoikilosis. All the affected individuals that we investigated were heterozygous with respect to a loss-of-function mutation in

LEMD3 (also called MAN1), which encodes an inner nuclear membrane protein. A somatic mutation in the second allele of LEMD3 could not be identified in fibroblasts from affected skin of an individual with BOS and an individual with melorheostosis. XMAN1, the Xenopus laevis ortholog, antagonizes BMP signaling during embryogenesis ${ }^{5}$. In this study, LEMD3 interacted with BMP and activin-TGF $\beta$ receptoractivated Smads and antagonized both signaling pathways in human cells.

Osteopoikilosis (OMIM 166700) is an autosomal dominant skeletal dysplasia characterized by a symmetric but unequal distribution of multiple hyperostotic areas in different parts of the skeleton (Fig. 1 $)^{6}$. These lesions, usually detected incidentally, represent foci of old remodeled bone with lamellar structure, either connected to adjacent trabeculae of spongy bone or attached to the subchondral cortex ${ }^{7}$. Osteopoikilosis can occur either as an isolated anomaly or in association with other abnormalities of skin and bone. BOS (OMIM 166700), an autosomal dominant disorder, refers to the association of osteopoikilosis with disseminated connective-tissue nevi. Both elastic-type nevi (juvenile elastoma) and collagen-type nevi (dermatofibrosis lenticularis disseminata) have been described in $\mathrm{BOS}^{8}$. Skin or bony lesions can be absent in some family members, whereas other relatives may have both ${ }^{9}$. The co-occurrence of osteopoikilosis and melorheostosis in the same family has been reported in a few instances ${ }^{2-4}$. Melorheostosis (OMIM 155950) is characterized by a 'flowing' (rheos) hyperostosis of the cortex of tubular bones. These lesions are usually asymmetric: they may involve only one limb or correspond to a particular sclerotome. They are often accompanied by abnormalities of adjacent soft tissues, such as joint contractures, sclerodermatous skin lesions, muscle atrophy, hemangiomas and lymphoedema ${ }^{10,11}$.
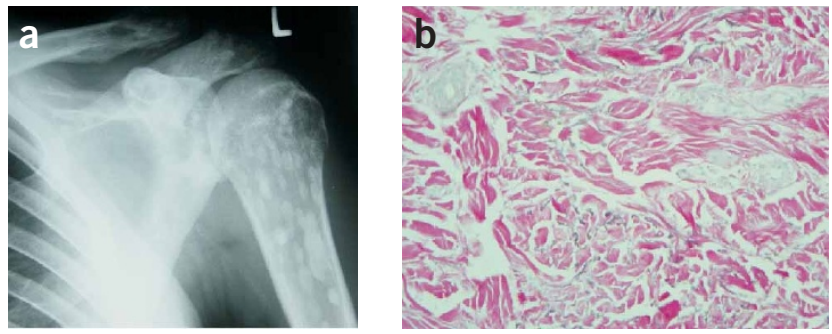

Figure 1 Osteopoikilosis lesions and elastic-type nevus in individual III-3 of family A. (a) Anteroposterior radiograph of the left shoulder showing multiple osteopoikilosis lesions, best visible in the left humerus. (b) Light micrograph of the elastic-type nevus stained with Van Gieson. Original magnification, $\times 100$. Thick and coarse collagen bundles with numerous broad and irregular elastic fibers are present in the mid-dermis.

${ }^{1}$ Center for Medical Genetics, Ghent University Hospital, Ghent, Belgium. ${ }^{2}$ Department of Developmental Biology, Flanders Interuniversity Institute for Biotechnology and Laboratory of Molecular Biology; and ${ }^{3}$ Center for Human Genetics, University of Leuven, Leuven, Belgium. ${ }^{4}$ Department of Orthopedic Surgery, Ghent University Hospital, Ghent, Belgium. ${ }^{5}$ Medical Genetics Service, Sainte-Justine Hospital and University of Montréal, Montréal, Canada. ${ }^{6}$ Department of Medical Genetics, University Hospital and University of Antwerp, Belgium. ${ }^{7}$ Genetic Health Services Victoria, Murdoch Childrens Research Institute, and University of Melbourne, Australia. ${ }^{8}$ Department of Radiology, University Hospital and University of Antwerp, Belgium. ${ }^{9}$ Department of Dermatology, Ghent University Hospital, Ghent, Belgium. Correspondence should be addressed to G.R.M. (geert.mortier@ugent.be). 


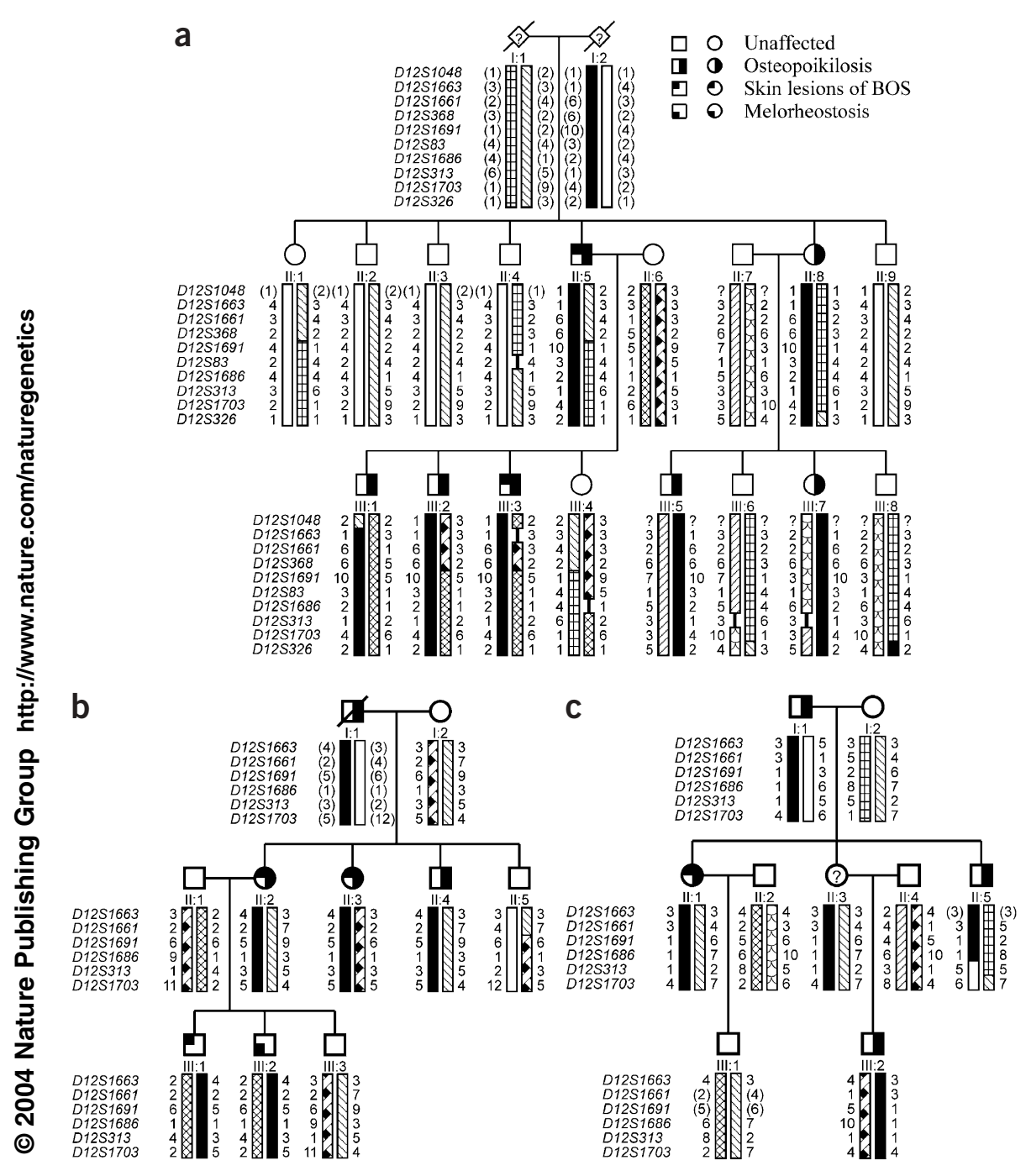

Figure 2 Pedigree structure and haplotypes of the three families with osteopoikilosis, family A (a), family B (b) and family C (c). Inferred alleles are shown in brackets. The haplotype cosegregating with the disorder is indicated with a black bar. osteopoikilosis. We hypothesized that this individual might have a microdeletion, resulting in the loss of several contiguous genes, including the gene mutated in osteopoikilosis. We investigated this individual for loss of heterozygosity in the candidate region on 12q12-12q14.3 and found loss of heterozygosity for marker D12S1686, located in the telomeric part of the interval. The testing of additional markers confirmed the presence of a microdeletion with a centromeric boundary at marker D12S329 (Fig. 3). The telomeric boundary of the microdeletion was defined at the single-nucleotide polymorphism tsc0527430 using the GeneChip Mapping $10 \mathrm{~K}$ Array (results for whole genome in Supplementary Fig. 1 online; results for chromosome 12 in Fig. 4c). We then tested more markers in the region of overlap between the microdeletion and the linkage interval in family $\mathrm{C}$, which allowed us to narrow the linkage interval and define a 3.07-Mb critical region for association with osteopoikilosis between marker D12S329 and $\mu$ SAT12.10. This region contains 23 known genes (National Center for Biotechnology Information genome viewer; Fig. 3).

Two of these genes, WIF1 (Wnt inhibitory factor 1) and LEMD3 (LEM domaincontaining 3), are good candidates for involvement in osteopoikilosis. WIF1 is involved in Wnt signaling, and LEMD3 functions in BMP signaling, two pathways important in bone development ${ }^{13,14}$. Mutation analysis of WIF1 did not identify any abnormalities in the affected individuals. Sequencing of LEMD3 identified loss-of-function mutations in all affected individuals of the three families and in three unrelated individuals with osteopoikilosis (Table 1 and Fig. 4e). The splicesite mutation in individual G03-2881 caused skipping of exon 6 , resulting in a frameshift Affected individuals may be asymptomatic or may complain of chronic pain in the affected limb ${ }^{12}$.

To unravel the genetic defect of osteopoikilosis, we started a genome-wide linkage analysis in family A (Fig. 2a). Screening of 400 markers with an average spacing of $10 \mathrm{cM}$ resulted in a significant positive lod score (3.744) for two consecutive markers on chromosome 12q13: D12S368 and D12S83. The results of the linkage analysis in the two other families, families B and C (Fig. 2b,c), were consistent with the linkage data obtained in family A. The centromeric boundary of the genetic interval was determined by a recombination event between D12S1048 and D12S1663 in individual III-1 (of family A). The telomeric boundary was defined by a recombination event between D12S1686 and D12S313 in individual II-5 (of family C), resulting in a candidate region of $23.55 \mathrm{cM}$ on chromosome 12q12-12q14.3. We calculated a combined maximum two-point lod score of 6.691 at $\theta=0$ for markers D12S1661 and D12S1691 (Supplementary Table 1 online).

The next step in the genetic analysis was the identification of individual G03-1858, who is affected with proportionate short stature, microcephaly, learning disabilities, ectopic kidneys and and premature stop codon in exon 7 at position 2,021 (Fig. 4d). The deletion of one of the LEMD3 alleles in individual G03-1858 was corroborated by fluorescence in situ hybridization (FISH) analysis with locus-specific probes (Fig. $4 \mathbf{b}$ ). Re-evaluation of the karyotype (550-band level) was suggestive of, but not conclusive for, the presence of a deletion in the 12q14-15 region (Fig. 4a).

Some reports have suggested that the asymmetric distribution of skin lesions in BOS and the segmental involvement usually observed in melorheostosis result from a somatic mutation ${ }^{4,15}$. To investigate this possibility, we took skin biopsy samples from two affected individuals, one from an elastic-type nevus in individual III-3 (of family A) with BOS and a second from a hard sclerodermic-like lesion in individual III-2 (of family B) with melorheostosis. Sequence analysis of LEMD3 on genomic DNA extracted from both skin lesions showed no evidence for an additional somatic mutation in LEMD3 (or 'second hit'). Analysis of intragenic polymorphisms showed no loss of heterozygosity or allelic imbalance and therefore excluded the possible existence of a partial gene deletion as a somatic mutation. In cDNA from normal and affected skin of the individual 


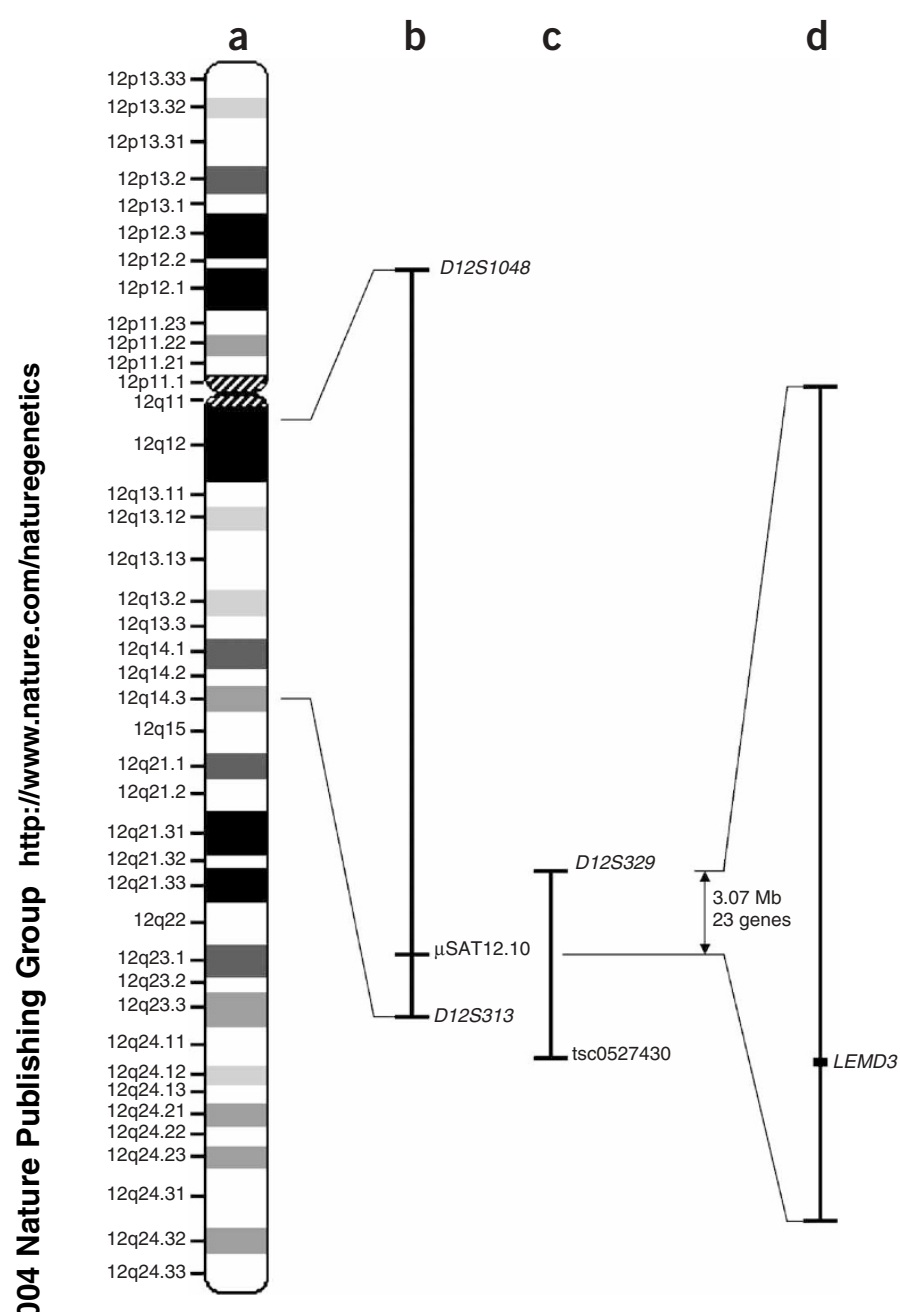

Figure 3 Ideogram of chromosome 12 showing the linkage interval, microdeletion and candidate region. (a) Ideogram of chromosome 12. (b) The 12q12-q14.3 linkage interval with indication of markers at the 20 boundaries. (c) The microdeletion in individual G03-1858 in relation to the linkage interval. The region of interest is shown on the right (d), with LEMD3 as the candidate gene.

with BOS, we found only the normal sequence, owing to nonsensemediated decay of mRNA from the abnormal allele. Because bone specimens were not available, we could not investigate the possibility that a somatic mutation in osteoblasts could explain the spotty occurrence of bone lesions.

LEMD3 is an integral protein of the inner nuclear membrane ${ }^{16}$. It contains a nucleoplasmic $\mathrm{N}$ - and C-terminal domain and two transmembrane segments ${ }^{16}$. The $\mathrm{N}$-terminal segment shares a conserved globular domain of $\sim 40$ amino acids with other inner nuclear membrane proteins, such as lamina-associated polypeptide 2 (LAP2) and emerin ${ }^{16}$. The $X$. laevis ortholog of LEMD3 (XMAN1) antagonizes BMP signaling. This antagonizing activity of XMAN1 resides in the C-terminal region that binds to Smad1, Smad5 and Smad8 (ref. 5).

To investigate whether LEMD3 interacts with BMP receptoractivated and TGF $\beta$ receptor-activated Smads, we carried out a yeast two-hybrid analysis using the C-terminal domain of LEMD3 as prey. This analysis identified interactions between the C-terminal domain of LEMD3 and the MH2 domains of Smad1 (BMP-specific) a

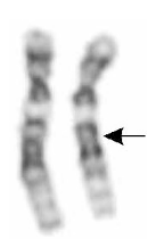

b

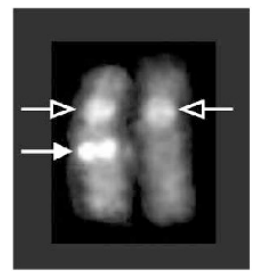

C

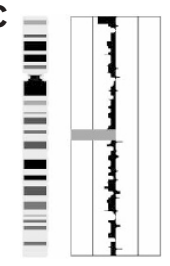

d

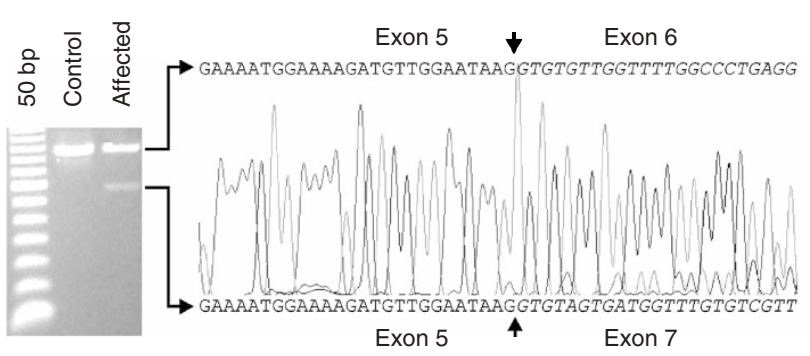

e

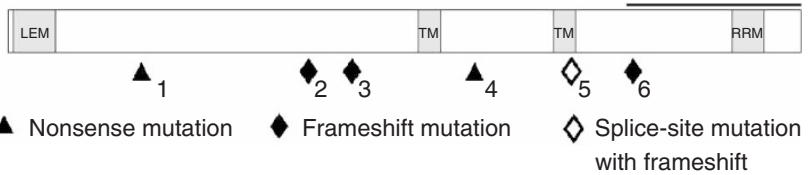

Figure 4 Overview of the cytogenetic and molecular defects found in affected individuals. (a) Partial karyotype from individual G03-1858 showing both chromosomes 12. The normal homolog is depicted on the left, and the homolog with the deletion (arrow) is shown on the right. (b) Metaphase FISH analysis with the BAC clone encompassing LEMD3 (RP11-30506; filled arrow) and the centromeric 12 probe (open arrow), showing a microdeletion on the right homolog. (c) Results from the GeneChip Mapping 10K Array analysis for chromosome 12 of individual G03-1858. An ideogram of chromosome 12 is shown on the left; meta-analysis significance of the genetic copy-number variation of each SNP against the reference mean is shown on the right. The region with large negative values (bar to the left) indicates the presence of a microdeletion. (d) Effect of the LEMD3 mutation in individual G03-2881. The electrophoretic analysis of an amplified cDNA fragment containing exons 4-8 shows the presence of a 146-bp shorter fragment as compared to the control. Partial nonsense-mediated decay is probably responsible for the weaker signal of this abnormal fragment. Sequence analysis shows skipping of exon 6 in the mutated allele (causing a frameshift with a premature stop codon in exon 7 at position 2,021; data not shown). (e) The positions of all LEMD3 mutations identified in this study are shown below the structure of LEMD3 (see also Table 1). Functional domains are indicated in gray: the LEM-containing $\mathrm{N}$-terminal domain, the two transmembrane (TM) domains and the C-terminal domain with the RNA-recognition motif (RRM) motif. The black horizontal bar indicates the Smad-interacting part and BMP-antagonizing portion (as shown in XMAN1; ref. 5) of the C-terminal domain.

and Smad2 (TGF $\beta$-specific), suggesting that LEMD3 is involved in both BMP and TGF $\beta$ signaling (Fig. 5a).

We further investigated the role of LEMD3 in both signaling pathways by overexpressing the protein in two different cell lines. In HEK293T cells, we measured the expression of several known target genes in basal conditions and after BMP4 stimulation by quantitative PCR (Q-PCR). Overexpression of LEMD3 reduced the capacity of BMP4 to upregulate Smad6, Smad7, Id2 and Id3 (Fig. 5b). In HepG2 cells, we measured the response of TGF $\beta$ using the activin-TGF $\beta$ responsive 3TP-Lux reporter in basal conditions and in the presence of a constitutively active receptor ALK4, which activates Smad2 and Smad3. Overexpression of LEMD3 reduced the ALK4-induced 
other genetic factors contribute to the presence and distribution of skin and bone lesions in these disorders. In addition, we found evidence that LEMD3 can antagonize both the BMP and TGF $\beta$ signaling pathway. These results are in accordance with previous studies showing that genetic defects in both signaling pathways can result in hyperostotic bone disorders. Loss-of-function mutations in SOST, encoding the extracellular BMP antagonist sclerostin, lead to sclerosteosis $^{17,18}$ (OMIM 269500), whereas activating missense mutations in TGFB1 can result in Camurati-Engelmann disease $\mathrm{e}^{19,20}$ of (OMIM 131300). Finally, increased signaling in the TGF $\beta$ pathway has been observed in other fibrotic skin disorders, such as scleroderma, and may therefore explain the skin lesions in individuals with BOS and melorheostosis ${ }^{21,22}$.

\section{METHODS}

Material from affected individuals. We obtained appropriate informed consent from all subjects involved in the study. Family A is a three-generation family of Belgian origin. All affected individuals had osteopoikilosis. Two affected individuals (II-5 and III-3) also had skin manifestations of BOS. In individual III-3, skin biopsy samples were taken from a connective-tissue nevus on the left thigh and from normal skin on the right thigh. Light microscopy showed more numerous elastin fibers in the middle and deep dermis on the affected side. These fibers had a slightly granular and thickened appearance (Fig. 1b). Ultrastructurally, the elastin fibers were much thicker and more 윽 numerous in the affected skin than in the normal skin. They had a welldeveloped amorphous matrix with delicate peripheral microfibrils. Results of 5 light microscopy and electron microscopy analysis were consistent with the diagnosis of connective-tissue nevus of the elastic-tissue type. Fibroblasts from both biopsies were cultured for molecular analysis.

Family B is another three-generation family of Belgian origin, previously reported ${ }^{2}$, illustrating the co-occurrence of osteopoikilosis and melorheostosis in one family. Family $\mathrm{C}$ is a three-generation British family with autosomal dominant osteopoikilosis and skin lesions reminiscent of BOS. Individual G031885 is an Australian with BOS. Individuals G03-2881 and G03-2882 have osteopoikilosis and are both of Belgian origin but are unrelated. Individual G G03-1858 had a history of prenatal and postnatal growth retardation and learning disability that was diagnosed as Russell-Silver syndrome in early (2) childhood. Investigations for hypertension in infancy revealed ectopic kidneys and aberrant renal arteries. Evaluation at the age of 16 years showed proportionate short stature with height of $131.5 \mathrm{~cm}$ (50th centile for a 9-y-old girl), weight of $31.8 \mathrm{~kg}$ (50th centile for a 10-y-old girl) and head circumference of $49 \mathrm{~cm}$ (50th centile for a 3-y-old girl). Physical examination revealed a subtly dysmorphic face with synophrys, mild hypertelorism, broad and high nasal bridge, micrognathia and maxillary overbite. In addition, a diffuse hyperpigmentation spot was noted on the left thigh. Osteopoikilosis lesions were found on skeletal survey.

Linkage analysis. We used all autosomal markers from the Applied Biosystems Linkage mapping set version 2 for a genome-wide linkage analysis. We used an improved protocol from the Centre National de Génotypage for pooling an average of four markers per PCR to carry out all reactions. Additional markers were taken from the Marshfield map or designed based on the simple-tandemrepeat finder in the University of California Santa Cruz genome browser. We carried out genotyping on an Applied Biosystems Prism 3100 Genetic Analyzer running Genemapper v2.0 software. We used the MLINK program of the LINKAGE software package ${ }^{23}$ to calculate two-point lod scores between the disease phenotype and each of the markers, assuming a dominant mode of inheritance with a penetrance of 0.95 and a disease allele frequency of 0.0001 . The frequency of most marker alleles was set to $1 / 8$. For markers with more than eight alleles in the pedigrees, the allele frequency was set to $1 / n$, where $n$ is the number of alleles.

Cytogenetic and FISH analysis. We carried out karyotyping in accordance with standard procedures. We analyzed metaphases at the 550-band level. We carried out FISH analysis as described ${ }^{24}$ using BAC clone RP11-30506 from the 12q14.3 region.
GeneChip Mapping 10K Array analysis. We analyzed DNA from individual G03-1858 using the GeneChip Mapping 10K Array (Affymetrix) as described ${ }^{25}$. We analyzed data with both the Affymetrix GCOS and GDAS software and the Affymetrix GeneChip Chromosome Copy Number Tool. We used copy number estimation (meta $P$ value) to identify the microdeletion, the boundaries of which were defined by loss of heterozygosity. Results were visualized with arrayCGHbase (B. Menten et al., unpublished data).

Sequence analysis. We amplified all exons by PCR using intronic primers and additional exonic primers for larger exons (primer sequences are available on request). We used a touchdown PCR program with an annealing temperature decreasing from $60{ }^{\circ} \mathrm{C}$ to $48{ }^{\circ} \mathrm{C}$ over 12 cycles, followed by 20 cycles with an annealing temperature of $48^{\circ} \mathrm{C}$. We sequenced PCR products using the BigDye v3.1 ET terminator cycle sequencing kit from Applied Biosystems. Sequencing reactions were loaded onto an Applied Biosystems Prism 3100 Genetic Analyzer and analyzed with Sequencing Analysis v3.7 and SeqScape v1.1 software (Applied Biosystems).

Yeast two-hybrid analysis. We cloned the C-terminal domain of human LEMD3, encoding amino acids $520-853$, as a PvuII-XhoI fragment into the SmaI and XhoI restriction sites of the prey vector pAct2 (Clontech). We cotransformed yeast strain AH109 with this vector or a positive control (SIP1) prey vector with different Smad bait vectors ${ }^{26}$, using the Yeastmaker yeast transformation system 2 (Clontech). We assayed activation of the gene MEL1 by interacting hybrid proteins in yeast on Trp-Leu drop-out medium containing the chromogenic substrate X- $\alpha$-Gal (Clontech).

Luciferase assay. We carried out transfections of HepG2 cells in triplicate in 24well plates using Fugene (Roche). Each well was transfected with a total amount of $500 \mathrm{ng}$ of plasmid DNA, including $50 \mathrm{ng}$ of 3TP-Lux reporter, $50 \mathrm{ng}$ of RSVpromoter-based lac $Z$ reporter and combinations of $50 \mathrm{ng}$ of the constitutively active ALK4 receptor with $100 \mathrm{ng}$ of LEMD3 or empty expression vector. We maintained a constant amount of DNA by adding pBluescript vector. Fortyeight hours after transfection, we assayed cell extracts for luciferase and $\beta$-galactosidase activities in accordance with the manufacturers' protocols (Promega and Clontech, respectively). We normalized the data by calculating the ratio of luciferase activity to $\beta$-galactosidase activity.

Q-PCR. We isolated RNA using RNeasy Mini Kit (Qiagen) and synthesized cDNA using SuperScript II Reverse Transcriptase Kit with random hexamer primers (Invitrogen) in a total volume of $20 \mu \mathrm{l}$. We used $5 \mu \mathrm{l}$ of cDNA (1:10 dilution) in combination with the Q-PCR Core Kit for SYBR Green I (Eurogentec) and $250 \mathrm{nM}$ gene-specific primers to carry out Q-PCR on a GeneAmp 5700 Sequence Detector (Applied Biosystems). The Q-PCR program consists of 40 cycles with $15 \mathrm{~s}$ at $95{ }^{\circ} \mathrm{C}$ and $1 \mathrm{~min}$ at $60{ }^{\circ} \mathrm{C}$, followed by a dissociation run to determine melting curves. We carried out all reactions in duplicate and normalized them to the geometric mean of three stable reference genes (GAPD, HPRT1 and YWHAZ) ${ }^{27}$.

To investigate the effect of LEMD3 on Smad signaling, we seeded HEK293T cells in 9-cm dishes and transfected them with a total of $2 \mu \mathrm{g}$ of LEMD3 plasmid DNA (provided by H. Worman; Department of Medicine, College of Physicians and Surgeons, Columbia University, New York, New York, USA) using Fugene (Roche). Twenty-four hours after transfection, cells were serumstarved for $4 \mathrm{~h}$ and then stimulated with $5 \mathrm{ng} \mathrm{ml}^{-1}$ of human recombinant BMP4 (R\&D Systems) for $1 \mathrm{~h}$ followed by RNA extraction.

In the screening for differentially expressed genes, we used fibroblasts from a control individual and from normal and affected skin of affected individual III-3 (of family A). We grew the cells in $13.5-\mathrm{cm}$ dishes to $80 \%$ confluency and then serum-starved them for $2 \mathrm{~h}$. We collected cells after $1 \mathrm{~h}$ of treatment with $1 \mathrm{ng} \mathrm{ml}^{-1}$ human recombinant TGF $\beta 1$ (R\&D Systems) or with $5 \mathrm{ng} \mathrm{ml}^{-1}$ of BMP4. Expression levels for all genes were determined in four independent experiments. Differential gene expression was considered significant when the difference was at least $50 \%$ and the $95 \%$ confidence interval of the mean expression ratio did not include 1 (equivalent with $P<0.05$ ).

We analyzed the following genes (their ID numbers in the RTPrimerDB database $^{28}$ are given in parentheses): GAPD (3), HPRT1 (5), YWHAZ (9), MMP2 (113), CTGF (596), COL1A1 (1089), COL3A1 (1090), COL5A1 (1091), FN1 (1092), ELN (1093), ID1 (1094), ID2 (1095), ID3 (1096), 
ID4 (1097), LEMD3 (1098), RUNX2 (1099), SMAD6 (1100), SMAD7 (1101) and SERPINE1 (1102).

Analysis of mutant LEMD3 constructs. We modified the wild-type LEMD3 construct (provided by H. Worman; Department of Medicine, College of Physicians and Surgeons, Columbia University, New York, New York, USA) to contain the $1185 \mathrm{dupT}, 1609 \mathrm{C} \rightarrow \mathrm{T}$ or $2154 \mathrm{dupA}$ mutation using the QuickChange Site-Directed Mutagenesis kit (Stratagene) in accordance with the manufacturer's instructions. We plated HEK293T cells at a density of $4 \times 10^{5}$ cells per well in Dulbecco's modified Eagle medium plus (Invitrogen) and transferred them to Opti-MEM I (Invitrogen) after overnight adherence. We transiently transfected cells with $2 \mu \mathrm{g}$ of each plasmid and $0.5 \mu \mathrm{g}$ of the $(\mathrm{CAGA})_{12}$ TGF $\beta$-responsive reporter construct ${ }^{29}$ in duplicate using Lipofectamine (Invitrogen). We also transfected cells with $20 \mathrm{ng}$ of pRL-TK (Promega) to correct for transfection efficiency. Cells were serum-starved for $7 \mathrm{~h}$ before stimulation with $7 \mathrm{ng} \mathrm{ml}^{-1}$ recombinant human TGF $\beta$ (R\&D systems). We lysed cells $24 \mathrm{~h}$ after transfection. We quantified activities of firefly and Renilla luciferase using the Dual-Luciferase Reporter Assay System (Promega).

URLs. Improved linkage analysis protocols from the Centre National de Génotypage are available at http://www.cng.fr/. The arrayCGHbase visualization tool and RTPrimerDB database are available at http://medgen.ugent.be/ arraycghbase/ and http://medgen.ugent.be/rtprimerdb/, respectively.

Note: Supplementary information is available on the Nature Genetics website.

\section{○ ACKNOWLEDGMENTS}

We thank the affected individuals and families for their interest and cooperation

M. Godfrey for critical review of the manuscript, P. Tylzanowski for suggestions and plasmid stocks and $\mathrm{H}$. Worman for the expression vector encoding human

O LEMD3. This study was supported, in part, by the Fund for Scientific Research, Flanders, with a mandate fundamental clinical research to P.D. and G.R.M.;

a research assistantship to P.C.M.V.; and research projects to F.S., S.J.T.V., W.V.H. and G.R.M. This study was also supported by an Interuniversity Attraction Pole

grant to W.V.H. and by the Fifth Framework of the specific research and

\. technological development program "Quality of Life and Management of Living

Resources" of the European Commission to J.H., A.D.P. and G.R.M. J.H. is funded

J by, and J.V. is a postdoctoral researcher with, the Institute for the Promotion

of Innovation by Science and Technology in Flanders. N.V.R. and K.J. are postdoctoral researchers of the Fund for Scientific Research, Flanders. The research at Flanders Interuniversity Institute for Biotechnology was supported by the Fund

(2) for Scientific Research, Flanders and the University of Leuven. O.P. is holder of a DWTC (Federal Services for Scientific, Technical and Cultural affairs) postdoctoral fellowship and is supported by the Interuniversity Attraction Pole Network. This

50 text presents research results of the Belgian program of Interuniversity Poles of attraction initiated by the Belgian State, Prime Minister's Office, Science Policy Programming. The scientific responsibility is assumed by the authors.

\section{COMPETING INTERESTS STATEMENT}

The authors declare that they have no competing financial interests.

Received 22 July; accepted 17 September 2004

Published online at http://www.nature.com/naturegenetics/

1. Hall, C.M. International nosology and classification of constitutional disorders of bone (2001). Am. J. Med. Genet. 113, 65-77 (2002).

2. Debeer, P., Pykels, E., Lammens, J., Devriendt, K. \& Fryns, J.P. Melorheostosis in a family with autosomal dominant osteopoikilosis: Report of a third family. Am. J. Med. Genet. 119A, 188-193 (2003).
3. Butkus, C.E., Michels, V.V., Lindor, N.M. \& Cooney, W.P. 3rd Melorheostosis in a patient with familial osteopoikilosis. Am. J. Med. Genet. 72, 43-46 (1997).

4. Nevin, N.C., Thomas, P.S., Davis, R.I. \& Cowie, G.H. Melorheostosis in a family with autosomal dominant osteopoikilosis. Am. J. Med. Genet. 82, 409-414 (1999).

5. Osada, S., Ohmori, S.Y. \& Taira, M. XMAN1, an inner nuclear membrane protein, antagonizes BMP signaling by interacting with Smad1 in Xenopus embryos. Development 130, 1783-1794 (2003).

6. Melnick, J.C. Osteopathia condensans disseminata (osteopoikilosis): study of a family of 4 generations. Am. J. Roentgenol. 82, 229-238 (1959).

7. Lagier, R., Mbakop, A. \& Bigler, A. Osteopoikilosis: a radiological and pathological study. Skeletal Radiol. 11, 161-168 (1984).

8. Ehrig, T. \& Cockerell, C.J. Buschke-Ollendorff syndrome: report of a case and interpretation of the clinical phenotype as a type 2 segmental manifestation of an autosomal dominant skin disease. J. Am. Acad. Dermatol. 49, 1163-1166 (2003).

9. Berlin, R., Hedensio, B., Lilja, B. \& Linder, L. Osteopoikilosis-a clinical and genetic study. Acta. Med. Scand. 181, 305-314 (1967).

10. Campbell, C.J., Papademetriou, T. \& Bonfiglio, M. Melorheostosis. A report of the clinical, roentgenographic, and pathological findings in fourteen cases. J. Bone Joint Surg. Am. 50, 1281-1304 (1968).

11. Rozencwaig, R., Wilson, M.R. \& McFarland, G.B. Jr. Melorheostosis. Am. J. Orthop. 26, 83-89 (1997).

12. Freyschmidt, J. Melorheostosis: a review of 23 cases. Eur. Radiol. 11, 474-479 (2001).

13. Gong, Y. et al. LDL receptor-related protein 5 (LRP5) affects bone accrual and eye development. Cell 107, 513-523 (2001).

14. Canalis, E., Economides, A.N. \& Gazzerro, E. Bone morphogenetic proteins, their antagonists, and the skeleton. Endocr. Rev. 24, 218-235 (2003).

15. Happle, R. Melorheostosis may originate as a type 2 segmental manifestation of osteopoikilosis. Am. J. Med. Genet. 125A, 221-223 (2004).

16. Lin, F. et al. MAN1, an inner nuclear membrane protein that shares the LEM domain with lamina-associated polypeptide 2 and emerin. J. Biol. Chem. 275, 4840-4847 (2000).

17. Balemans, W. et al. Increased bone density in sclerosteosis is due to the deficiency of a novel secreted protein (SOST). Hum. Mol. Genet. 10, 537-543 (2001).

18. Brunkow, M.E. et al. Bone dysplasia sclerosteosis results from loss of the SOST gene product, a novel cystine knot-containing protein. Am. J. Hum. Genet. 68, 577-589 (2001).

19. Janssens, K. et al. Mutations in the gene encoding the latency-associated peptide of TGF- $\beta 1$ cause Camurati-Engelmann disease. Nat. Genet. 26, 273-275 (2000).

20. Kinoshita, A. et al. Domain-specific mutations in TGF- $\beta 1$ result in Camurati-Engelmann disease. Nat. Genet. 26, 19-20 (2000).

21. Mori, Y., Chen, S.J. \& Varga, J. Expression and regulation of intracellular SMAD signaling in scleroderma skin fibroblasts. Arthritis Rheum. 48, 1964-1978 (2003).

22. Asano, Y., Ihn, H., Yamane, K., Kubo, M. \& Tamaki, K. Impaired Smad7-Smurfmediated negative regulation of TGF- $\beta$ signaling in scleroderma fibroblasts. J. Clin. Invest. 113, 253-264 (2004).

23. Lathrop, G.M. \& Lalouel, J.M. Easy calculations of lod scores and genetic risks on small computers. Am. J. Hum. Genet. 36, 460-465 (1984).

24. Van Roy, N. et al. 1;17 translocations and other chromosome 17 rearrangements in human primary neuroblastoma tumors and cell lines. Genes Chromosomes Cancer 10, 103-114 (1994).

25. Matsuzaki, H. et al. Parallel genotyping of over 10,000 SNPs using a one-primer assay on a high-density oligonucleotide array. Genome Res. 14, 414-425 (2004).

26. Verschueren, $\mathrm{K}$. et al. SIP1, a novel zinc finger/homeodomain repressor, interacts with Smad proteins and binds to $5^{\prime}$-CACCT sequences in candidate target genes. J. Biol. Chem. 274, 20489-20498 (1999).

27. Vandesompele, J. et al. Accurate normalization of real-time quantitative RT-PCR data by geometric averaging of multiple internal control genes. Genome Biol. 3 RESEARCH0034 (2002).

28. Pattyn, F., Speleman, F., De Paepe, A. \& Vandesompele, J. RTPrimerDB: the real-time PCR primer and probe database. Nucleic Acids Res. 31, 122-123 (2003).

29. Dennler, S. et al. Direct binding of Smad3 and Smad4 to critical TGF $\beta$-inducible elements in the promoter of human plasminogen activator inhibitor-type 1 gene. EMBO J. 17, 3091-3100 (1998). 